\title{
THE MÖBIUS-POMPEÏU METRIC PROPERTY
}

BRANKO J. MALEŠEVIĆ

Received 17 September 2004; Revised 22 November 2004; Accepted 27 June 2005

We consider an extension of Möbius-Pompeïu theorem of the elementary geometry over metric spaces. We specially take into consideration Ptolemaic metric spaces.

Copyright (C 2006 Branko J. Malešević. This is an open access article distributed under the Creative Commons Attribution License, which permits unrestricted use, distribution, and reproduction in any medium, provided the original work is properly cited.

\section{The Möbius-Pompeïu theorem and metric spaces}

In this paper we consider the following statement of elementary geometry $[2,3]$.

Theorem 1.1 (Möbius-Pompeïu). Let $A B C$ be an equilateral triangle and $M$ any point in its plane. Then segments $M A, M B$, and $M C$ are sides of a triangle.

Let us consider an analogous problem for the metric space $(X, d)$ with at least four points. Let $A, B, C \in X$ be three fixed points. Then, for the point $M \in X$, we suppose that a triangle can be formed from the distances $d_{1}=d(M, A), d_{2}=d(M, B)$, and $d_{3}=d(M, C)$ if and only if the following conjunction of inequalities is true:

$$
d_{1}+d_{2}-d_{3} \geq 0, \quad d_{2}+d_{3}-d_{1} \geq 0, \quad d_{3}+d_{1}-d_{2} \geq 0
$$

If in conjunction (1.1) at least one equality is true, then we suppose that a degenerative triangle can be formed. If in (1.1) sharp inequalities are true:

$$
d_{1}+d_{2}-d_{3}>0, \quad d_{2}+d_{3}-d_{1}>0, \quad d_{3}+d_{1}-d_{2}>0,
$$

then we suppose that a nondegenerative triangle can be formed. In this case, for the point $M$, for which the conjunction (1.2) is true, we define that the point has Möbius-Pompeïu metric property. The main subject of this paper is to determine points $M$ which do not have Möbius-Pompeïu metric property, that is, these points which fulfill the following disjunction of the inequalities:

$$
d_{1}+d_{2}-d_{3} \leq 0, \quad d_{2}+d_{3}-d_{1} \leq 0, \quad \text { or } \quad d_{3}+d_{1}-d_{2} \leq 0 .
$$

Hindawi Publishing Corporation Journal of Inequalities and Applications Volume 2006, Article ID 83206, Pages 1-9 DOI 10.1155/JIA/2006/83206 
Let us notice that the point $M \in\{A, B, C\}$ does not have Möbius-Pompeïu metric property. Thus in consideration which follows, we assume that the metric space $(X, d)$ has at least four points.

\section{Ptolemaic metric spaces}

A metric space $(X, d)$ is called a Ptolemaic metric space if Ptolemaic inequality holds:

$$
d\left(x_{1}, x_{2}\right) d\left(x_{3}, x_{4}\right) \leq d\left(x_{2}, x_{4}\right) d\left(x_{1}, x_{3}\right)+d\left(x_{1}, x_{4}\right) d\left(x_{2}, x_{3}\right)
$$

for every $x_{1}, x_{2}, x_{3}, x_{4} \in X[1]$. A normed space $(X,|\cdot|)$ is a Ptolemaic normed space if metric space $(X, d)$ is Ptolemaic with the distance $d(x, y)=|x-y|$. Let us notice that the following lemma is true [1].

Lemma 2.1. A normed space is Ptolemaic if and only if it is an inner product space.

We give two basic examples of Ptolemaic spaces [1].

Example 2.2. $\left(1^{0}\right)$ The space $\mathbb{R}^{n}$ with the Euclidean metric $d(x, y)=|x-y|$ is a Ptolemaic metric space.

$\left(2^{0}\right)$ The space $\mathbb{R}^{n}$ with the chordal metric on the unit Riemann sphere $\bar{d}(x, y)=2 \mid x-$ $y \mid /\left(\sqrt{1+|x|^{2}} \sqrt{1+|y|^{2}}\right)$ is a Ptolemaic metric space.

We will illustrate the following considerations with the previous examples of Ptolemaic metric spaces in the case of dimension $n=2$.

\section{The main results}

Let $(X, d)$ be a metric space. Let us fix three points $A, B, C \in X$ and form distances:

$$
a=d(B, C), \quad b=d(C, A), \quad c=d(A, B) .
$$

For any point $M \in X$ let us form distances:

$$
d_{1}=d(M, A), \quad d_{2}=d(M, B), \quad d_{3}=d(M, C) .
$$

Inequality $d_{2}+d_{3} \leq d_{1}$. Let us determine a set of $M$ points of metric spaces $X$ for which the following inequality is true:

$$
d_{2}+d_{3} \leq d_{1}
$$

Let us form two functions:

$$
\begin{gathered}
\alpha_{1}=\alpha_{1}(M)=4 d_{2}^{2} d_{3}^{2}-\left(d_{1}^{2}-\left(d_{2}^{2}+d_{3}^{2}\right)\right)^{2}, \\
\beta_{1}=\beta_{1}(M)=d_{2}^{2}+d_{3}^{2}-d_{1}^{2} .
\end{gathered}
$$

Lemma 3.1. For points $A, B$, and $C$, inequality $\alpha_{1} \leq 0$ is true.

Proof. For point $A, d_{1}=0$ and $\alpha_{1}=-\left(c^{2}-b^{2}\right)^{2} \leq 0$ are true. Similarly, the previous inequality is true for the points $B$ and $C$. 
Example 3.2. Let vertices $A, B, C$ of the triangle $A B C$ in the plane $\mathbb{R}^{2}$ be given by coordinates $A\left(a_{1}, b_{1}\right), B\left(a_{2}, b_{2}\right), C\left(a_{3}, b_{3}\right)$, and let $M(x, y)$ be any point in its plane.

$\left(1^{0}\right)$ Let us in the plane $\mathbb{R}^{2}$ use Euclidean metric $d$. Let us specify the forms of the terms $\alpha_{1}$ and $\beta_{1}$ which correspond to functions (3.4), respectively. It is true that

$$
\alpha_{1}=k\left(x^{2}+y^{2}\right)^{2}+\left(A_{1} x+B_{1} y\right)\left(x^{2}+y^{2}\right)+C_{1} x^{2}+D_{1} x y+E_{1} y^{2}+F_{1} x+G_{1} y+H_{1}
$$

for some coefficients $k, A_{1}, B_{1}, C_{1}, D_{1}, E_{1}, F_{1}, G_{1}, H_{1} \in \mathbb{R}(k=3)$. Equality $\alpha_{1}=0$ determines the algebraic curve of the fourth order. By inequality $\alpha_{1}<0$, we determine the interior of the previous curve. Also, it is true that

$$
\beta_{1}=A_{2}\left(x^{2}+y^{2}\right)+B_{2} x+C_{2} y+D_{2}
$$

for some coefficients $A_{2}, B_{2}, C_{2}, D_{2} \in \mathbb{R}\left(A_{2}=1\right)$. If $B_{2}^{2}+C_{2}^{2}>4 D_{2}$, the equality $\beta_{1}=0$ is possible and determines the circle. Then by inequality $\beta_{1}<0$, we determine the interior of the circle.

$\left(2^{0}\right)$ Let us in the plane $\mathbb{R}^{2}$ use chordal metric $\bar{d}$. Let us specify the forms of the terms $\bar{\alpha}_{1}$ and $\bar{\beta}_{1}$ which correspond to functions (3.4), respectively. It is true that

$$
\bar{\alpha}_{1}=\frac{\bar{k}\left(x^{2}+y^{2}\right)^{2}+\left(\bar{A}_{1} x+\bar{B}_{1} y\right)\left(x^{2}+y^{2}\right)+\bar{C}_{1} x^{2}+\bar{D}_{1} x y+\bar{E}_{1} y^{2}+\bar{F}_{1} x+\bar{G}_{1} y+\bar{H}_{1}}{\left(1+x^{2}+y^{2}\right)^{2}\left(1+a_{1}^{2}+b_{1}^{2}\right)^{2}\left(1+a_{2}^{2}+b_{2}^{2}\right)^{2}\left(1+a_{3}^{2}+b_{3}^{2}\right)^{2}}
$$

for some coefficients $\bar{k}, \bar{A}_{1}, \bar{B}_{1}, \bar{C}_{1}, \bar{D}_{1}, \bar{E}_{1}, \bar{F}_{1}, \bar{G}_{1}, \bar{H}_{1} \in \mathbb{R}$. If $\bar{k} \neq 0$, the equality $\bar{\alpha}_{1}=0$ determines the algebraic curve of the fourth order. Then by inequality $\bar{\alpha}_{1}<0$, we determine the interior of the previous curve. Also, it is true that

$$
\bar{\beta}_{1}=\frac{\bar{A}_{2}\left(x^{2}+y^{2}\right)+\bar{B}_{2} x+\bar{C}_{2} y+\bar{D}_{2}}{\left(1+x^{2}+y^{2}\right)\left(1+a_{1}^{2}+b_{1}^{2}\right)\left(1+a_{2}^{2}+b_{2}^{2}\right)\left(1+a_{3}^{2}+b_{3}^{2}\right)}
$$

for some coefficients $\bar{A}_{2}, \bar{B}_{2}, \bar{C}_{2}, \bar{D}_{2} \in \mathbb{R}$. If $\bar{A}_{2} \neq 0$ and $\bar{B}_{2}^{2}+\bar{C}_{2}^{2}>4 \bar{A}_{2} \bar{D}_{2}$, the equality $\bar{\beta}_{1}=$ 0 is possible and determines the circle. Then by the inequality $\bar{\beta}_{1}<0$, we determine the interior of the circle.

Further, let us notice that for the function $\alpha_{1}$,

$$
\alpha_{1}=\left(d_{2}+d_{3}-d_{1}\right)\left(d_{3}+d_{1}-d_{2}\right)\left(d_{1}+d_{2}-d_{3}\right)\left(d_{1}+d_{2}+d_{3}\right) .
$$

According to (3.9), the equality $\alpha_{1}=0$ is equivalent with the union of equalities

$$
\begin{gathered}
\alpha_{1}^{(1)}=d_{2}+d_{3}-d_{1}=0, \\
\alpha_{1}^{(2)}=d_{3}+d_{1}-d_{2}=0, \\
\alpha_{1}^{(3)}=d_{1}+d_{2}-d_{3}=0 .
\end{gathered}
$$

Subject to our further consideration is an inequality $\alpha_{1}^{(1)} \leq 0$.

Lemma 3.3. $\left(1^{0}\right)$ For the point $B, d_{2}+d_{3} \leq d_{1}$ if and only if $c \geq a$.

$\left(2^{0}\right)$ For the point $C, d_{2}+d_{3} \leq d_{1}$ if and only if $b \geq a$. 
Remark 3.4. If $a>b, c$, then for the points $B$ and $C, \alpha_{1} \leq 0$ and $\alpha_{1}^{(1)}>0$.

Lemma 3.5. If for point $M, d_{2}+d_{3} \leq d_{1}$, then the following inequalities hold:

$$
\begin{aligned}
& d_{1}+d_{2} \geq d_{3}, \quad \text { where the equality is true for } M=B, a=c, \\
& d_{3}+d_{1} \geq d_{2}, \quad \text { where the equality is true for } M=C, a=b .
\end{aligned}
$$

Proof. It is true that

$$
\left(d_{1}\right)+d_{2}-d_{3} \geq\left(d_{2}+d_{3}\right)+d_{2}-d_{3}=2 d_{2} \geq 0 .
$$

Hence, the inequality (3.11) follows. Thus, the equality is true only if $M=B\left(d_{2}=0\right)$ and $a=c$. Analogously, it is true that

$$
d_{3}+\left(d_{1}\right)-d_{2} \geq d_{3}+\left(d_{2}+d_{3}\right)-d_{2}=2 d_{3} \geq 0 .
$$

Hence, the inequality (3.12) follows. Thus, the equality is true only if $M=C\left(d_{3}=0\right)$ and $a=b$.

Lemma 3.6. ( $\left(^{0}\right)$ If the point $M$ fulfills $d_{2}+d_{3} \leq d_{1}$, then the following implication is true:

$$
\alpha_{1} \leq 0 \Longrightarrow \beta_{1} \leq 0
$$

$\left(2^{0}\right)$ If the point $M$ fulfills $d_{3}+d_{1} \leq d_{2}$ or $d_{1}+d_{2} \leq d_{3}$, then the following implication is true:

$$
\alpha_{1} \leq 0 \Longrightarrow \beta_{1} \geq 0 \text {. }
$$

Proof. The implications (3.15) and (3.16) have the same assumptions:

$$
\alpha_{1}=4 d_{2}^{2} d_{3}^{2}-\left(d_{1}^{2}-d_{2}^{2}-d_{3}^{2}\right)^{2}=\left(2 d_{2} d_{3}-d_{1}^{2}+d_{2}^{2}+d_{3}^{2}\right)\left(2 d_{2} d_{3}+d_{1}^{2}-d_{2}^{2}-d_{3}^{2}\right) \leq 0,
$$

which follow if the conjunction

$$
\left(2 d_{2} d_{3}-d_{1}^{2}+d_{2}^{2}+d_{3}^{2}\right) \leq 0, \quad\left(2 d_{2} d_{3}+d_{1}^{2}-d_{2}^{2}-d_{3}^{2}\right) \geq 0
$$

or

$$
\left(2 d_{2} d_{3}-d_{1}^{2}+d_{2}^{2}+d_{3}^{2}\right) \geq 0, \quad\left(2 d_{2} d_{3}+d_{1}^{2}-d_{2}^{2}-d_{3}^{2}\right) \leq 0
$$

is true

$\left(1^{0}\right)$ Let $d_{2}+d_{3} \leq d_{1}$ be true. For $M=B$ or $M=C$, implication (3.15) is directly verified. Especially for $M=B$ and $a=c$ or for $M=C$ and $a=b$, the equality $\beta_{1}=0$ is true. Let us assume that $M \neq B, C$ and let us assume that $\alpha_{1} \leq 0$ in (3.15) is true. On the basis of $d_{2}+d_{3} \leq d_{1}$, according to Lemma 3.5, it follows that $d_{1}+d_{2}>d_{3}$ and $d_{3}+d_{1}>d_{2}$. Therefore,

$$
\begin{gathered}
2 d_{2} d_{3}-d_{1}^{2}+d_{2}^{2}+d_{3}^{2}=\left(d_{2}+d_{3}\right)^{2}-d_{1}^{2} \leq 0, \\
2 d_{2} d_{3}+d_{1}^{2}-d_{2}^{3}-d_{3}^{2}=\left(d_{1}-d_{2}+d_{3}\right)\left(d_{1}+d_{2}-d_{3}\right)>0 .
\end{gathered}
$$


From (3.20), we can conclude that the conjunction (3.18) is true and conjunction (3.19) is not true. From the conjunction (3.18), it follows that $d_{1}^{2}-d_{2}^{2}-d_{3}^{2} \geq 2 d_{2} d_{3}>d_{2}^{2}+d_{3}^{2}-d_{1}^{2}$, and from there, $d_{1}^{2}>d_{2}^{2}+d_{3}^{2}$, that is, $\beta_{1}<0$.

$\left(2^{0}\right)$ Let $d_{3}+d_{1} \leq d_{2}$ be true. For $M=B$ or $M=C$, implication (3.16) is directly verified. Especially for $M=B$ and $a=c$ or for $M=C$ and $a=b$, the equality $\beta_{1}=0$ is true. Let us assume that $M \neq B, C$ and let us assume that $\alpha_{1} \leq 0$ in (3.16) is true. On the basis of $d_{3}+d_{1} \leq d_{2}$, according to the lemma analogous to Lemma 3.5, it follows that $d_{2}+d_{3}>d_{1}$ and $d_{1}+d_{2}>d_{3}$. Therefore

$$
\begin{gathered}
2 d_{2} d_{3}-d_{1}^{2}+d_{2}^{2}+d_{3}^{2}=\left(d_{2}+d_{3}\right)^{2}-d_{1}^{2}>0, \\
2 d_{2} d_{3}+d_{1}^{2}-d_{2}^{3}-d_{3}^{2}=\left(d_{1}-d_{2}+d_{3}\right)\left(d_{1}+d_{2}-d_{3}\right) \leq 0 .
\end{gathered}
$$

From (3.21), we can conclude that conjunction (3.19) is true and conjunction (3.18) is not true. From conjunction (3.19), it follows that $d_{2}^{2}+d_{3}^{2}-d_{1}^{2} \geq 2 d_{2} d_{3}>d_{1}^{2}-d_{2}^{2}-d_{3}^{2}$ and therefore, $d_{2}^{2}+d_{3}^{2}>d_{1}^{2}$, that is, $\beta_{1}>0$. The implication (3.16) is similarly verified in the case of the inequality $d_{1}+d_{2} \leq d_{3}$.

Lemma 3.7. In the metric space $X$, the condition $d_{2}+d_{3} \leq d_{1}$ is equivalent to the conjunctions $\alpha_{1} \leq 0$ and $\beta_{1} \leq 0$.

Proof. $(\Rightarrow)$ Let for the point $M$ the condition $d_{2}+d_{3} \leq d_{1}$ be true. On the basis of the equality (3.9) and on the basis of Lemma 3.5, it follows that $\alpha_{1} \leq 0$. Therefore, on the basis of Lemma 3.6, it follows that $\beta_{1} \leq 0$.

$(\Leftarrow)$ Let for the point $M$ conjunctions $\alpha_{1} \leq 0$ and $\beta_{1} \leq 0$ be true. Then from the conjunction

$$
\alpha_{1}=\left(d_{2}+d_{3}-d_{1}\right)\left(d_{2}+d_{3}+d_{1}\right)\left(2 d_{2} d_{3}-\beta_{1}\right) \leq 0, \quad \beta_{1} \leq 0
$$

it follows the condition $d_{2}+d_{3} \leq d_{1}$.

Lemma 3.8. In Ptolemaic metric space $X$, an inequality $\alpha_{1}^{(1)} \leq 0$ is true if and only if $b \geq a$ or $c \geq a$.

Proof. On the basis of Lemma 3.3, if $a \leq c$, then for the point $B$, we have $\alpha_{1}^{(1)}=a-c \leq 0$, or if $a \leq b$, then for the point $C$, we have $\alpha_{1}^{(1)}=b-a \leq 0$. Conversely, let $a>b, c$ be true. Let $M \in X \backslash\{A, B, C\}$ be any point. Then on the basis of the Ptolemaic inequality

$$
c \cdot d_{3}+b \cdot d_{2} \geq a \cdot d_{1}
$$

and the assumption $a>b, c$, we can conclude that

$$
(c-a) d_{3}+(b-a) d_{2}+a\left(d_{2}+d_{3}-d_{1}\right) \geq 0 \Rightarrow \alpha_{1}^{(1)}=d_{2}+d_{3}-d_{1}>0 .
$$

By contraposition the statement follows.

On the basis of the previous lemmas, we can conclude that the following theorem is true. 
Theorem 3.9. In the metric space X, a point $M$ fulfills $\alpha_{1}^{(1)}=d_{2}+d_{3}-d_{1} \leq 0$ if and only if $\alpha_{1} \leq 0$ and $\beta_{1} \leq 0$ are true. In Ptolemaic metric space $X$, the set of these points $M$ is nonempty if and only if

$$
b \geq a \quad \text { or } \quad c \geq a .
$$

Inequalities $d_{2}+d_{3} \leq d_{1}, d_{3}+d_{1} \leq d_{2}, d_{1}+d_{2} \leq d_{3}$. Let us determine a set of points $M$ in (Ptolemaic) metric spaces for which some inequalities in (1.3) are true. With respect to point $A$, we formed functions (3.4). Next, with respect to point $B$, let us form the functions

$$
\begin{gathered}
\alpha_{2}=\alpha_{2}(M)=4 d_{3}^{2} d_{1}^{2}-\left(d_{2}^{2}-\left(d_{3}^{2}+d_{1}^{2}\right)\right)^{2}, \\
\beta_{2}=\beta_{2}(M)=d_{3}^{2}+d_{1}^{2}-d_{2}^{2},
\end{gathered}
$$

and with respect to $C$ point, let us form the functions

$$
\begin{gathered}
\alpha_{3}=\alpha_{3}(M)=4 d_{1}^{2} d_{2}^{2}-\left(d_{3}^{2}-\left(d_{1}^{3}+d_{2}^{2}\right)\right)^{2}, \\
\beta_{3}=\beta_{3}(M)=d_{1}^{2}+d_{2}^{2}-d_{3}^{2} .
\end{gathered}
$$

The following equality $\alpha_{1}=\alpha_{2}=\alpha_{3}$ is true. Analogously to Theorem 3.9, we can conclude that the following theorems are true.

Theorem 3.10. In the metric space $X$, point $M$ fulfills $\alpha_{1}^{(2)}=d_{3}+d_{1}-d_{2} \leq 0$ if and only if $\alpha_{1} \leq 0$ and $\beta_{2} \leq 0$ are true. In Ptolemaic metric space $X$, the set of these points $M$ is nonempty if and only if

$$
c \geq b \quad \text { or } \quad a \geq b \text {. }
$$

Theorem 3.11. In the metric space $X$, point $M$ fulfills $\alpha_{1}^{(3)}=d_{1}+d_{2}-d_{3} \leq 0$ if and only if $\alpha_{1} \leq 0$ and $\beta_{3} \leq 0$ are true. In Ptolemaic metric space $X$, the set of these points $M$ is nonempty if and only if

$$
a \geq c \quad \text { or } \quad b \geq c .
$$

For (Ptolemaic) metric space $X$, the set of the points $M$ with Möbius-Pompeïu metric property fulfill a conjunction

$$
\alpha_{1}^{(1)}>0, \quad \alpha_{1}^{(2)}>0, \quad \alpha_{1}^{(3)}>0 .
$$

Using Theorems 3.9, 3.10, and 3.11, we can determine when some inequalities in (3.30) are not true.

Finally, in the following example, let us illustrate a set of points in $\mathbb{R}^{2}$ with MöbiusPompeïu metric property, with respect to three fixed points $A, B, C \in \mathbb{R}^{2}$, if we use metrics $d$ and $\bar{d}$ from Example 2.2.

Example 3.12. $\left(1^{0}\right)$ Let in the plane $\mathbb{R}^{2}$ the Euclidean metric $d$ be used. By Figure 3.1, we illustrate the case of the triangle $A B C$ for which $a>c>b$ is true. Then $\alpha_{1}^{(1)}>0$ is 


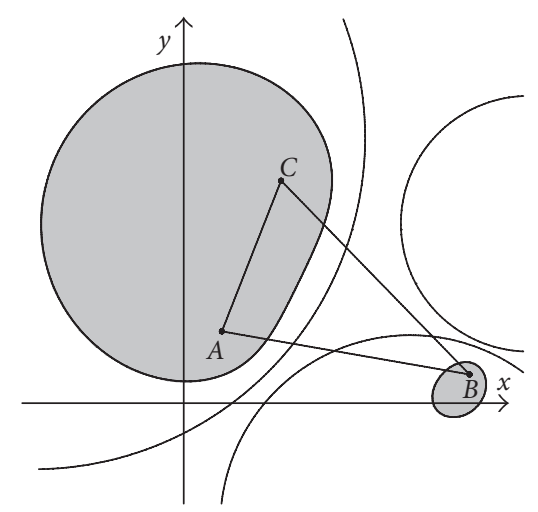

Figure 3.1

true (the curve $\alpha_{1}^{(1)}=0$, on the basis of Theorem 3.9, has empty interior and border), otherwise the curves $\alpha_{1}^{(2)}=0, \alpha_{1}^{(3)}=0$ have nonempty interior and border. We can form a nondegenerative triangle from the remaining points.

In the case of the equilateral triangle $A B C$, the curves $\alpha_{1}^{(1)}=0, \alpha_{1}^{(2)}=0$, and $\alpha_{1}^{(3)}=0$ transform onto the (smaller) arcs $\hat{B C}, \hat{C A}$, and $\hat{A B}$ of the circumcircle. Hence, we have Möbius-Pompeïu theorem in the following form: for equilateral triangle $A B C$, the set of points $M$ in the plane, such that from distances $d_{1}=d(M, A), d_{2}=d(M, B)$, and $d_{3}=$ $d(M, C)$ one can form a degenerative triangle, is circumcircle; from the other points in the plane we can form nondegenerative triangle.

$\left(2^{0}\right)$ Let in the plane $\mathbb{R}^{2}$ the chordal metric $\bar{d}$ is used. Let $A, B, C \in \mathscr{S} \backslash\{(0,0,1)\}$ be points on the unit Riemann sphere $\mathscr{S}$, with uniquely determined projections:

$$
A^{\prime}=\mathscr{P}^{-1}(A)=a_{1}+b_{1} i, B^{\prime}=\mathscr{P}^{-1}(B)=a_{2}+b_{2} i, C^{\prime}=\mathscr{P}^{-1}(C)=a_{3}+b_{3} i \in \mathbb{C},
$$

with inversely stereographical projection from the north pole:

$$
\mathscr{P}^{-1}=\mathscr{P}^{-1}(x, y, z)=\left(\frac{x}{1-z}\right)+\left(\frac{y}{1-z}\right) i: \mathscr{Y} \backslash\{(0,0,1)\} \longrightarrow \mathbb{C}
$$

Through points $A, B, C$ on the Riemann sphere, let us set great circles (Figure 3.2). In the complex plane we uniquely determine images of great circles as corresponding circles through points $A^{\prime}, B^{\prime}, C^{\prime}$ (Figure 3.3). By Figure 3.3 we illustrate the case of points $A^{\prime}, B^{\prime}, C^{\prime}$ for which $\bar{b}>\bar{c}>\bar{a}$ and $\bar{k} \neq 0$ are true. Then $\bar{\alpha}_{1}^{(2)}>0$ (the curve $\bar{\alpha}_{1}^{(2)}=0$, on the basis of Theorem 3.10, has empty interior and border), otherwise curves $\bar{\alpha}_{1}^{(1)}=0$, $\bar{\alpha}_{1}^{(3)}=0$ have nonempty interior and border. From the remaining points, we can form a nondegenerative triangle.

Let us consider the case when $A, B, C$ are chordally equidistantly arranged points on the Riemann sphere $\mathscr{S}$. Then the set of points $M$ on the Riemann sphere, being such that 


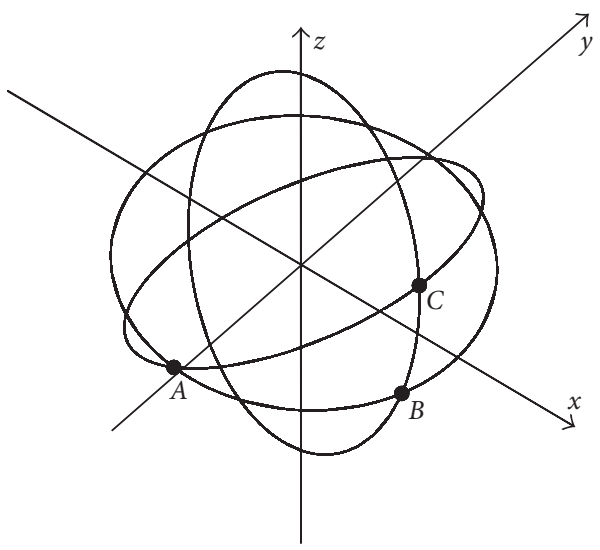

Figure 3.2

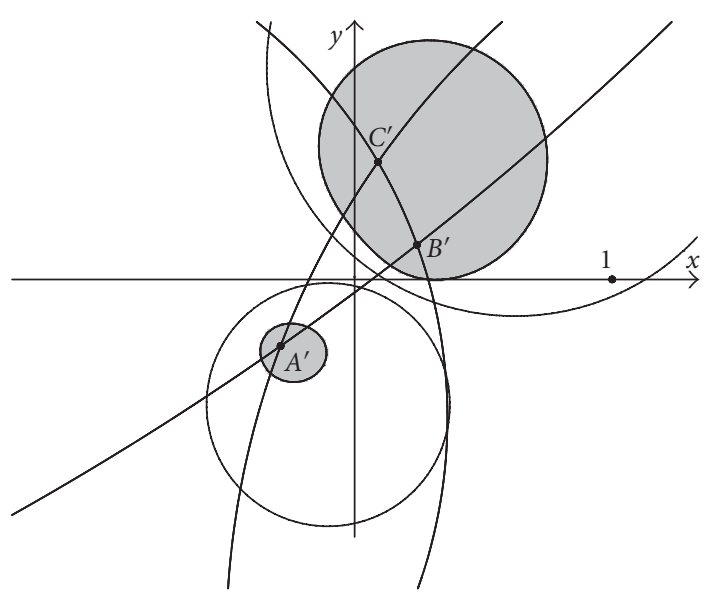

Figure 3.3

from chordal distances $\bar{d}_{1}=\bar{d}(M, A), \bar{d}_{2}=\bar{d}(M, B)$, and $\bar{d}_{3}=\bar{d}(M, C)$ one can form a degenerative triangle, is circumcircle; from other points on the Riemann sphere one can form a nondegenerative triangle. Using inverse stereographical projection $\mathscr{P}^{-1}$ we can conclude that analogous statement in complex plane $\mathbb{C}$ is valid if we use chordal metric $\bar{d}$.

\section{Acknowledgment}

The research is partially supported by the MNTRS, Serbia \& Montenegro, Grant No. 144020. 


\section{References}

[1] P. A. Hästö, A new weighted metric: the relative metric. I, Journal of Mathematical Analysis and Applications 274 (2002), no. 1, 38-58.

[2] D. S. Mitrinović, J. E. Pečarić, and V. Volenec, History, variations and generalizations of the Möbius-Neuberg theorem and the Möbius-Pompeiu theorem, Bulletin Mathématique de la Société des Sciences Mathématiques de la République Socialiste de Roumanie. Nouvelle Série 31(79) (1987), no. 1, 25-38.

[3] _ Recent advances in geometric inequalities, The Möbius-Neuberg and the MöbiusPompeïu Theorems, chapter 13, Kluwer Academic, Dordrecht, 1989, pp. 385-400.

Branko J. Malešević: Faculty of Electrical Engineering, University of Belgrade, Bulevar Kralja Aleksandra 73, Belgrade, Serbia \& Montenegro

E-mail address:malesevic@etf.bg.ac.yu 